\title{
Smoking cessation and lung cancer screening: new perspectives from the SCALE project
}

\author{
Filippo Lococo ${ }^{1}$, Giuseppe Cardillo ${ }^{2}$, Giulia Veronesi ${ }^{3}$ \\ ${ }^{1}$ Unit of Thoracic Surgery, Azienda Unità Sanitaria Locale-IRCCS di Reggio Emilia, Italy; ${ }^{2}$ Unit of Thoracic Surgery, Azienda Ospedaliera San \\ Camillo Forlanini, Rome, Italy; ${ }^{3}$ Unit of Thoracic Surgery, Humanitas Cancer Center, Milan, Italy \\ Correspondence to: Filippo Lococo, MD. Unit of Thoracic Surgery, IRCCS- Arcispedale Santa Maria Nuova, via Risorgimento 80, 42100 Reggio \\ Emilia, Italy. Email: filippo_lococo@yahoo.it. \\ Provenance: This is an invited Editorial commissioned by the Executive Editor-in-Chief Jianxing He (Department of Cardiothoracic Surgery, The \\ First Affiliated Hospital of Guangzhou Medical University, Guangzhou, China). \\ Comment on: Joseph AM, Rothman AJ, Almirall D, et al. Lung Cancer Screening and Smoking Cessation Clinical Trials. SCALE (Smoking Cessation \\ within the Context of Lung Cancer Screening) Collaboration. Am J Respir Crit Care Med 2018;197:172-82.
}

Submitted Aug 30, 2018. Accepted for publication Sep 20, 2018.

doi: $10.21037 /$ jtd.2018.09.122

View this article at: http://dx.doi.org/10.21037/jtd.2018.09.122

In the last few years, tobacco smoking has shown steady rates, stabilizing at round 1.1 billion smokers since 2000. All in all, $12 \%$ of people 30 years or older die because of smoking (1), a fact which establishes smoking as a major cause of premature and preventable morbidity and mortality (2). Onset of smoking age also impacts mortality. According to a Japanese Six Prefecture Study, both male and female smokers who started smoking when aged $<20$ years showed death rates slightly higher than those related to smokers who started smoking at older ages (3). Large studies carried out in different countries $(4,5)$ have examined smoking consequences on mortality in smoking populations who started smoking in early adult life and did not quit. Among these studies, the British Doctors' Study (6) and Framingham Heart Study reported (7) that in middle-aged smokers (between 30 and 69), the mortality rate was 2 or 3 times higher than the rate of people who never smoked, thus leading to an approximately 10-year reduction in life span. Therefore, the 2012 WHO global report (8) estimated that death rates related to tobacco smoking would increase from about 5 million in 2010 to more than 10 million in a few decades, due to current young smokers reaching middle and old age. With such an alarming prediction, smoking cessation clearly stands as a public health priority. Tumor incidence, which is directly related to cigarette smoking (lung cancer in primis), should decrease if a more smoking-averse attitude is adopted by the population.
In accordance with several scientific societies engaged in lung-cancer treatment, the US Preventive Services Task Force (USPSTF) issued recommendations suggesting low-dose CT (LDCT) screening for high-risk people due to age and smoking history (Grade B) (9), in addition to implementing large scale efficient and effective systems of smoking cessation. However, studies and data supporting the feasibility and effectiveness of smoking cessation treatment remain scarce, especially within the framework of lung cancer screening (LCS).

In this context, the "Smoking Cessation within the Context of Lung Cancer Screening" (SCALE) project, a collaborative group composed of eight LCS trials and reported on in the American fournal of Respiratory and Critical Care Medicine (AJRCCM) (10) by Joseph and colleagues, deserves particular attention. It aims at standardizing both the method and design of smoking cessation programs within LCS trials. Funded by a 2015 National Cancer institute (NCI) initiative, the trials included different study designs such as traditional RCTs, cluster randomized control designs, sequential multiple assignment randomized trials (SMART), factorial experimental designs and modeling of LCS outcomes including potential health benefits of smoking cessation treatment. The SCALE project aimed at facilitating data sharing, peer feedback and maximizing clinical trial findings.

Authors reviewed and analyzed different factors affecting 
tobacco treatment outcomes, such as clinical workflow, participant eligibility criteria, screening indication (baseline or annual repeat screen), assessment content, interest in quitting smoking, treatment delivery method and dose (10).

According to US regulations covering 2015-activated LCS, the Center for Medicare and Medicaid Services (CMS) (11) set up strict criteria for LDCT LCS, such as the provision of smoking cessation counselling to patients prior to screening and reimbursement of imaging charges when offering smoking cessation counseling. Nevertheless, such advisable measures were problematic, as many LCS sites' delivery of tobacco cessation care faced significant barriers including inadequate patient interest, lack of staff training and reimbursement complexities related to services for smoking cessation.

Smoking cessation programs should consider the fact that patient participation rates in LCS hardly represent the general smoking population. Indeed, according to Joseph and colleagues' analysis (10), the participants are older than the general smoking population (aged more than 55 years or older), and they show more frequent co-morbidities and are more likely to be heavy, longstanding smokers. Even though there is a body of evidence suggesting that older smokers could be resistant to smoking cessation interventions (12), other trials have stressed that older smokers show deep interest in quitting smoking (13).

The difference between the LCS population and the population on whole is also apparent in the level of personal drive toward smoking cessation. Heavy smokers who are interested in LCS programs have already demonstrated intense motivation towards achieving better health. Smoking cessation still represents a very challenging goal for the entire population, due to the reality of the actual motivation of those concerned, and the lack of an accurate and sophisticated smoking cessation program. Therefore, it can be reasonably believed that subjects undergoing LCS programs are optimal candidates for smoking cessation programs and can finally succeed in quitting smoking.

In addition, screening outcomes might improve the impact of measures for smoking cessation in screening participants. Among several research teams $(14,15)$, we (16) documented the effect of CT-results on participants. Indeed, when small undetermined pulmonary nodules are detected, a "re-call" CT scan represents a strong "teachable moment", defined as a health event, that motivates patients to adopt risk-reducing health behaviors. Several other factors might play an important role, such as a concern for a "positive test", fear of a lung cancer diagnosis, and physicians who warn about lung cancer and smoking risks.

As has been previously reported by Joseph (10), the combination of LCS and smoking withdrawal led to a maximum reduction in NLST mortality (17). With similar results, Pastorino and colleagues studied the consequences of smoking cessation on overall mortality of participants in repeated LDCT screening (3,381 cases) for many years in the context of a MILD trial (18). They reported a 39\% drop of mortality in patients who quit smoking compared to current smokers and former ones. According to their analysis, the effect of quitting smoking in reducing overall mortality turned out to be 3 to 5 times higher than that recorded at earlier detection in the MILD Trial.

In the light of these results, we might speculate optimistically on the remarkable future impact of the SCALE project in terms of reduced mortality in the populations involved. It is hoped that smoking cessation programs are going to increasingly represent a cornerstone in lung cancer prevention.

The development of effective smoking cessation programs within screening projects turns out to be even more important in Europe (not more difficult!) than in the USA if we consider that according to the European LCS trails $(19,20)$, rates of active smokers in the screened population are substantially higher ( $70 \%$ to $80 \%)$ (18) compared to the enrolled population in the US screened cohorts (around 50\%) (17), with higher potential impact of this initiative on mortality.

The 8 ongoing SCALE projects are testing different combinations of smoking cessation interventions, thus offering opportunities of "tailored" smoking cessation strategies with the aim to increase survivorship rates from the rates found in screening-only groups.

By incorporating positive and negative screening results, and coordinating smoking cessation treatment with clinical events associated with LCS, new and promising opportunities will emerge to reduce lung cancer morbidity and mortality through an effective smoking cessation treatment.

\section{Acknowledgements}

We thank Dr. Daniela Masi (Reggio Emilia AUSL-IRCCS) for her support in English editing.

\section{Footnote}

Conflicts of Interest: The authors have no conflicts of interest to declare. 


\section{References}

1. Available online: http://www.who.int/news-room/factsheets/detail/tobacco

2. Jha P, Peto R. Global effects of smoking, of quitting, and of taxing tobacco. N Engl J Med 2014;370:60-8.

3. Uno F, Ishikawa $S$, Nakamura $Y$, et al. Smoking and risk of all-cause mortality: the Jichi Medical School (JMS) Cohort Study. J Epidemiol 2005;15:173.

4. Jha P, Ramasundarahettige C, Landsman V, et al. 21stCentury hazards of smoking and benefits of cessation in the United States. N Engl J Med 2013;368:341-50.

5. Sakata R, McGale P, Grant EJ, et al. Impact of smoking on mortality and life expectancy in Japanese smokers: a prospective cohort study. BMJ 2012;345:e7093.

6. Boyle P. Tobacco smoking and the British doctors' cohort. Br J Cancer 2005;92:419-20.

7. Tindle HA, Stevenson Duncan M, et al. Lifetime Smoking History and Risk of Lung Cancer: Results From the Framingham Heart Study. J Natl Cancer Inst 2018;110:1201-7.

8. Peto R, Lopez AD. The future worldwide health effects of current smoking patterns. In: Koop E, Pearson CE, Schwarz MR, editors. Critical issues in global health. San Francisco: Jossey-Bass, 2001:154-61.

9. U.S. Preventive Services Task Force. Screening for Lung Cancer: Current Recommendation, 2013.

10. Joseph AM, Rothman AJ, Almirall D, et al. Lung Cancer Screening and Smoking Cessation Clinical Trials. SCALE (Smoking Cessation within the Context of Lung Cancer Screening) Collaboration. Am J Respir Crit Care Med 2018;197:172-82.

11. Available online: https://www.cms.gov/medicare-coveragedatabase/details/nca-decision-memo.aspx?NCAId=274\&N caName=Screening+for+Lung+Cancer+with+Low+Dose+ Computed+Tomography+(LDCT) $\&$ TimeFrame=7 \&Doc Type=All \&bc=AQAAIAAAAgAAAA $\% 3 \mathrm{~d} \% 3 \mathrm{~d} \&$

12. Messer K, Trinidad DR, Al-Delaimy WK, et al. Smoking cessation rates in the United States: A comparison of young adult and older smokers. Am J Public Health 2008;98:317-22.

13. Cataldo JK. High-risk older smokers' perceptions, attitudes, and beliefs about lung cancer screening. Cancer Med 2016;5:753-9.

14. Munshi V, McMahon P. Importance of Smoking Cessation in a Lung Cancer Screening Program. Curr Surg Rep $2013 ; 1$.

15. van der Aalst CM, van den Bergh KA, Willemsen MC, et al. Lung cancer screening and smoking abstinence: 2 year follow-up data from the Dutch-Belgian randomised controlled lung cancer screening trial. Thorax 2010;65:600-5.

16. Filippo L, Principe R, Cesario A, et al. Smoking cessation intervention within the framework of a lung cancer screening program: preliminary results and clinical perspectives from the "Cosmos-II" Trial. Lung 2015;193:147-9.

17. National Lung Screening Trial Research Team, Aberle DR, Adams AM, et al. Reduced lung-cancer mortality with low-dose computed tomographic screening. N Engl J Med 2011;365:395-409.

18. Pastorino U, Rossi M, Rosato V, et al. Annual or biennial CTscreening versus observation in heavy smokers: 5 -year results of the MILD trial. Eur J Cancer Prev 2012;21:308-15.

19. Veronesi G, Maisonneuve P, Rampinelli C, et al. Computed tomography screening for lung cancer: results of ten years of annual screening and validation of cosmos prediction model. Lung Cancer 2013;82:426-30.

20. Wille MM, Dirksen A, Ashraf H, et al. Results of the Randomized Danish Lung Cancer Screening Trial with Focus on High-Risk Profiling. Am J Respir Crit Care Med 2016;193:542-51.

(English Language Editor: John Gray, AME Publishing Company)
Cite this article as: Lococo F, Cardillo G, Veronesi G. Smoking cessation and lung cancer screening: new perspectives from the SCALE project. J Thorac Dis 2018;10(Suppl 33):S3999-S4001. doi: 10.21037/jtd.2018.09.122 